\title{
The Application of the Unascertained Rational Number In Calculating the Gravitational Acceleration
}

\author{
Hong-Yan $\mathrm{Li}^{1 \text {, a }}$ Ning Cai ${ }^{1, b^{*}}$ Zong-Yuan Tan ${ }^{1, c}$ Jian Zhou ${ }^{1, d}$ \\ ${ }^{1}$ College of Electrical Engineering, Northwest Minzu University, Lanzhou, China \\ c1403959623@qq.com, bcaining91@tsinghua.org.cn, ctanzongyuansmile@163.com \\ zhoujianfrank@126.com
}

*The corresponding author: Cai Ning (caining91@tsinghua.org.cn)

Keywords:Unascertained rational; Gravity acceleration; Application

\begin{abstract}
This article starts with solving the actual problems, raises the definition of unascertained rational numbers and the unascertained rational number method in the calculation of the gravitational acceleration, which implements the unascertained rational number in the application of the practical problems. It is more scientific to calculate the acceleration of gravity by using the method of unascertained rational number according to the calculation result.
\end{abstract}

\section{Introduction}

Professor Wang Guangyuan of the Harbin institute of architecture and engineering, a member of the Chinese academy of engineering have published the article "unascertained information and its mathematical processing" in the 1990, and reveal the first page of unascertained mathematics [1]. Under the guidance of professor Wang, professor Wu Heqin who belongs to construction in Hebei institute of science and technology gives accurate definition of unascertained number to express and process of unascertained information, and conceive the fundamental framework of unascertained mathematics $[2,3]$. After many peer's efforts, it has completed the basic content of the framework and has opened up pathways and methods of research that are different from randomness, ambiguity and grayness. These related contents are called unascertained math [4].

Unascertained mathematics is proposed to solve practical problems, but from the application point of view, unascertained rational number is the most basic and most important part. Here are some examples:

The weight of a built building.

The duration of a major earthquake in history, the location of the epicenter, etc.

To find Laowang, he is not at home, his whereabouts.

Above these questions, the objective thing itself is certain, but it could not exactly answer problems for policy makers, so in the minds of decision makers, it is uncertain. This subjective and cognitive uncertainty known as unascertained. It is different to the randomness which is only with respect to the things that will be happen in the future, and also is different to the fact that because it is impossible to give a certain definition and evaluation standard to form fuzzification in the certain domain, and also is not equal to the grayness. It has more information than the amount of gray information forming the amount of information that is grasped by decision makers, which is another uncertainty that is provided by objective reality.

At present, in the uncertain information that have been recognized by people, their uncertainty can be summarized as randomness, ambiguity, grayness and uncertainty [5,6]. The randomness is expressed and processed by the probability in mathematics. The ambiguity is expressed and processed by fuzzy mathematics. The gray matter is expressed and processed by the gray system theory [7]. However, uncertainty have not yet expressed and addressed by mature mathematical tools. The unascertained meaning is that whether or not the object itself is certain or uncertain, it is not known to the decision maker that it is partially known and unknown [8]. Hence, there is a great deal of uniformity in the nature 
of the unascertained quantity and the ash content, and they all know some of them and not all of them, but apart from that, there are important differences. The difference is that the known portion of the gray quantity is less than the known part of the unascertained quantity. In other words, in terms of its mathematical performance, the unascertained number increases the amount of "certainty" comparing to the grey number $[9,10]$. For example, if you only know that a number falls on the interval $[a, b]$, then this is an interval grey number, and if you also know that it has some distribution in the area, that's an unascertained number. Therefore, although both are means in "incomplete information mathematical processing", it is two different mathematical processing means, which have formed their own different mathematical content and system.

\section{The definition of unascertained rational number}

If one conducts a measurement, when it measures $\mathrm{n}$ times, measured values by size order is $x_{1}, x_{2}, \cdots, x_{k}$, and the number of occurrences of them in turn to $n_{1}, n_{2}, \cdots, n_{k}$, we call $\left[\left[x_{1}, x_{k}\right], \varphi(x)\right]$ as the distribution density type of the unascertained rational number, which is referred to as "the unascertained rational number", and $\varphi(x)$ is expressed as follows:

$$
\varphi(x)=\left\{\begin{array}{l}
\frac{n_{i}}{n}, x=x_{i}(i=1,2, \cdots, k) \\
0, \text { others }
\end{array}\right.
$$

Let $\left[x_{1}, x_{k}\right]$ be the value range of the unidentified rational number, $\varphi(x)$ is the reliability distribution density function for it. Obviously, $\sum_{i=1}^{k} n_{i}=n$ and a certain degree of $x_{0}$ in the $x_{i}$ of the credibility should be $\frac{n_{i}}{n}$ and $\sum_{i=1}^{k} \frac{n_{i}}{n}=1$.

\section{Model building and application}

When process information with unascertained rational number, we could generally conduct by the following steps.

Step1: Ask questions to determine whether the issue under discussion is a matter of unascertained rationality.

Step2: Analysis of influence factors to determine the main influence factors.

Step3: Establish an unascertained mathematical model.

Step4: Solve the model

There are many factors affecting the acceleration of gravity, such as the length of the pendulum, the cycle, the friction of the single point of contact, the air resistance, the geographical position and the stratigraphic structure, etc. However, the main factors are pendulum length and cycle, and other factors are secondary which could be negligible in the measurement process. One knows by physics that $\mathrm{g}=4 \pi^{2} L / T^{2}$, where $\mathrm{g}$ is the gravitational acceleration, $\mathrm{L}$ is the pendulum length and $\mathrm{T}$ is the pendulum cycle.

The pendulum length $\mathrm{L}$ and the period $\mathrm{T}$ were measured ten times respectively. Measuring $\mathrm{L}$ is $0.98 \mathrm{~m}$ twice, $1 \mathrm{~m}$ five times, $1.02 \mathrm{~m}$ three times, then the pendulum length $\mathrm{L}$ is $0.98 \mathrm{~m}, 1 \mathrm{~m}$ and $1.02 \mathrm{~m}$ and its credibility is $0.2,0.5$ and 0.3 , respectively. Measured T 1.7s twice, 2.0s six times, $2.2 \mathrm{~s}$ twice, then the cycle is $1.7 \mathrm{~s}, 2.0 \mathrm{~s}$ and $2.2 \mathrm{~s}$ and its credibility is $0.2,0.6$ and 0.2 , respectively. The pendulum length and cycle can be represented by unascertained rational number:

$$
L=\left[[0.98,1.02], \varphi_{L}(x)\right]
$$




$$
\begin{gathered}
\varphi_{L}(x)=\left\{\begin{array}{c}
0.2, x=0.98 \\
0.5, x=1.00 \\
0.3, x=1.02 \\
0, \text { others }
\end{array}\right. \\
T=\left[[1.7,2.2], \varphi_{T}(x)\right] \\
\varphi_{T}(x)=\left\{\begin{array}{cc}
0.2, x=1.7 \\
0.6, x=2.0 \\
0.2, x=2.2 \\
0, \text { others }
\end{array}\right.
\end{gathered}
$$

The possible value of the bounded matrix of $T^{2}$ :

$\begin{array}{llll}1.7 & 2.89 & 3.4 & 3.74\end{array}$

$\begin{array}{llll}2.0 & 3.4 & 4.0 & 4.4\end{array}$

$\begin{array}{llll}2.2 & 3.74 & 4.4 & 4.84\end{array}$

$\begin{array}{llll}-\quad & 1.7 & 2.0 & 2.2\end{array}$

Corresponding confidence with edge product matrix:

$\begin{array}{cccc}0.2 & 0.04 & 0.12 & 0.0 \\ 0.6 & 0.12 & 0.36 & 0.12 \\ 0.2 & 0.04 & 0.12 & 0.04\end{array}$

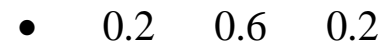

The elements of the possible value product matrix of $T^{2}$ are arranged in rows from small to large: $2.89,3.4,3.74,4.0,4.4,4.84$, and thus get closed [2.89, 4.84].

We align the $T^{2}$ confidence product matrix with the elements in its possible value product matrix in a row: $0.04,0.24,0.08,0.36,0.24,0.04$.

Can be obtained:

$$
\begin{gathered}
T^{2}=T \bullet T=\left[[2.89,4.84], \varphi_{T^{2}}(x)\right] \\
\varphi_{T^{2}}(x)=\left\{\begin{array}{l}
0.04, x=2.89 \\
0.24, x=3.4 \\
0.08, x=3.74 \\
0.36, x=4.0 \\
0.24, x=4.4 \\
0.04, x=4.84 \\
0, \text { others }
\end{array}\right.
\end{gathered}
$$

Take $\pi=3.14$, the value of $L$ and $T$ into the formulag $=4 \pi^{2} L / T^{2}$, for $4 \times 3.14 \times 3.14 L$ get

$$
4 \pi^{2} L=\left[[38.69,40.268], \varphi^{\prime}(x)\right]
$$




$$
\varphi^{\prime}(x)=\left\{\begin{array}{c}
0.2, x=38.68 \\
0.5, x=39.48 \\
0.3, x=40.268 \\
0, \text { others }
\end{array}\right.
$$

The possible values of $4 \pi^{2} L$ and $T^{2}$ with edge quotient matrix:

$\begin{array}{ccccccc}38.69 & 13.39 & 11.38 & 10.34 & 9.67 & 8.79 & 7.99 \\ 39.48 & 13.66 & 11.61 & 10.56 & 9.87 & 8.97 & 8.16 \\ 40.268 & 13.93 & 11.83 & 10.77 & 10.07 & 9.15 & 8.32 \\ \div & 2.89 & 3.4 & 3.74 & 4.0 & 4.4 & 4.84\end{array}$

$4 \pi^{2} L$ and $T^{2}$ credibility with the edge product matrix:

$\begin{array}{lllllll}0.2 & 0.008 & 0.048 & 0.016 & 0.072 & 0.048 & 0.008\end{array}$

$\begin{array}{lllllll}0.5 & 0.02 & 0.12 & 0.4 & 0.18 & 0.12 & 0.02\end{array}$

$\begin{array}{lllllll}0.3 & 0.012 & 0.072 & 0.024 & 0.108 & 0.072 & 0.012\end{array}$

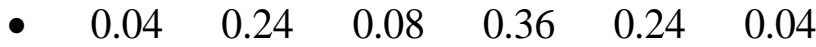

Finally, we obtain the results as follows:

$$
\begin{gathered}
g=4 \pi^{2} L / T^{2}=[[7.99,13.93], \varphi(x)] \\
\varphi(x)=\left\{\begin{array}{c}
0.008, x=7.99 \\
0.02, x=8.16 \\
0.012, x=8.32 \\
0.048, x=0.12 \\
0.12, x=8.97 \\
0.072, x=9.15 \\
0.072, x=9.67 \\
0.18, x=9.87 \\
0.108, x=10.07 \\
0.016, x=10.34 \\
0.04, x=10.56 \\
0.024, x=10.7 \\
0.048, x=11.38 \\
0.12, x=11.61 \\
0.072, x=11.84 \\
0.008, x=13.39 \\
0.02, x=13.66 \\
0.012, x=13.93 \\
0, \text { others }
\end{array}\right.
\end{gathered}
$$

From the above results, it can be seen that: 
When $\mathrm{x}=9.87$, the maximum of the credibility is 0.18 , which as a point confidence, 0.18 is the largest. Since the acceleration of gravity in a region is a certain value, the acceleration of gravity should be around that number.

Take $\mathrm{x}=9.87$ down three numbers to 8.97 and take three numbers up to 10.56 . At this point, the probability on the credibility of the value of the gravitational acceleration falls on the interval $[8.97,10.56]$ more than $60 \%$. It is considered that the desired gravity acceleration value falls on the interval, and it is also in line with reality that one regards the mean value of the seven numbers as the gravitational acceleration value of certain area.

\section{Conclusion}

We divide the things in the objective world into two categories. One is something that has occurred or is already present and there is clearly no randomness here and no matter how complicated the matter is, it is certain. When the decision makers are dealing with such things, they can not see it as definite, but must be treated as an indeterminate thing. This kind of problems are the objects which are addressed by undetermined rational number. The other is something that does not happen, and if all possible test results are known, this is a random test. Probability statistics is a powerful tool for studying randomized trials and dealing with random information. Even if the random phenomenon, when the statistical regularity is not very clear and difficult to determine the distribution function, using the methods of probability statistical to deal with the problems is not necessarily able to get satisfactory results. hence, it is the objects that will be exactly addressed by unascertained rational number.

In the general systems, there are always two kinds of factors. One is the state factor, which is objective; the other is the behavioral factor, which is subjective. The state factors are the objective foundation for forming behavioral factors, and the behavioral factors make the state factor to be manifested. If the systems with behavioral factors are called the general system, the general systems are actually unascertained systems. The information of such systems is entirely expressed and addressed by the tool that is unascertained rational number.

The unascertained rational number is the most basic, the simplest, the most widely used, and easily use, which is a generalization of the real number and can portray and express the objective reality "unascertained quantity" for avoiding only using a real number to indicate the amount of information missing and distortion of the defects. The maximal characteristic of this method is to retain all known information and directly participate in the quantitative operation, thus the accumulation of errors can be minimized. In addition to the original data, there is no artificial hypothesis and is furthest loyal to the given data.

\section{References}

[1] Y. Q. Guan, Z. J. Ji, L. Zhang and L. Wang: Int. J. Robust Nonlin. Control, DOI: 10.1002/rnc.3798.

[2] Z. J. Ji and H. S. Yu: IEEE Trans. Cybernet., DOI: 10.1109/TCYB.2016.2549034.

[3] N. Cai, J. W. Cao, H. Y. Ma and C. X. Wang: Arab J Sci Eng, Vol. 39 (2014) No.3, pp. 2427-2434.

[4] H. F. Ke, Y. G. Chen and H. M. Du: Chinese Control and Decision Conference (Qingdao, China, May 23-25, 2015), 2015, pp.5293-5296.

[5] J. X. Xi, M. He, H. Liu and J. F. Zheng: J Franklin Inst, Vol. 353 (2016) No.16, pp.4074-4090.

[6] N. Cai, M. He, Q. Wu and M. J. Khan: J. Syst. Sci. Complexity, DOI: 10.1007/s11424-017-6273-7.

[7] Q. J. Gu and Y. X. Sai: Joint International Conference on Modelling and Simulation (Wuxi, China, June 04-06, 2010), 2010, pp.79-82.

[8] Z. J. Ji, H. Lin and H. S. Yu: IEEE Trans. Automat. Control, Vol. 60 (2015) No.3, pp.781-786.

[9] N. Cai, M. J. Khan: Int. J. Control Automat. Syst., Vol. 15 (2017) No.1, pp.169-177.

[10] N. Cai, C. Diao and M. J. Khan: Complexity, 4978613, 2017. 